\title{
APRENDIZAJE UBICUO DE LOS NUEVOS APRENDICES Y BRECHA DIGITAL FORMATIVA
}

\section{UBIQUITOUS LEARNING OF NEW STUDENTS AND EDUCATIONAL DIGITAL DIVIDE}

\author{
Dra. María del Pilar Quicios García ${ }^{1}$ \\ pquicios@edu.uned.es
Dra. Isabel Ortega Sánchez ${ }^{1}$ iortega@edu.uned.es \\ Dra. Ma Paz Trillo Miravalles ${ }^{2}$ \\ mptrillo@edu.uned.es
}
Universidad Nacional de Educación a Distancia. Facultad de Educación.
${ }^{(1)}$ Departamento de Teoría de la Educación y Pedagogía Social.
${ }^{(2)}$ Departamento de Métodos y Diagnóstico en Educación I.
C/Juan del Rosal, 14. 28040, Madrid.

La crítica que reiteradamente ha recibido la escuela ha sido su inmovilidad metodológica. Impartición de contenidos emergentes con didácticas obsoletas. Este reproche puede haber sido superado. El siglo XX introdujo las TICen la escuela generando otros tipos de aprendizaje y otro tipo de estudiantes: los nuevos aprendices. Algunos de ellos, ahora son universitarios y gestionan sus aprendizajes de manera ubicua a través del uso de dispositivos móviles. Otros no los gestionan así. Esta dualidad metodológica origina una brecha digital formativa. Este artículo muestra los lugares en los que algunos nuevos aprendices universitarios utilizan dispositivos móviles para desarrollar su u-learning.

Palabras clave:Aprendizaje ubicuo, entornos virtuales de aprendizaje, enseñanza universitaria.

The criticism that has repeatedly received the school has been methodological immobility. Delivery of emerging content with outdated teaching. This criticism may have been exceeded. The twentieth century introduced ICTs in school generating other types of learning and other students: new apprentices. Some of them are now college and managet heir learning ubiquitously throught he use of mobile devices. Others do not managewell. This duality creates a formative digital divide.This article shows someplaces new college learners use mobile devices to develop u-learning.

Keywords:Ubiquitous learning, virtual learning environments, university education. 


\section{Introducción.}

En este artículo se extractan los resultados más relevantes del proyecto I+D MICINN EDU 2010-17420 que versan sobre los lugares en los que algunos estudiantes universitarios utilizan, con fines formativos, un conjunto de dispositivos móviles para desarrollar una nueva modalidad de aprendizaje: el aprendizaje ubicuo o u-learning.

Los dispositivos de los que se ofrecen resultados son los terminales que han mostrado, en el estudio realizado, mayor frecuencia de utilización entre los nuevos aprendices universitarios. Estos dispositivos son: el ordenador portátil, el teléfono móvil (no inteligente y smartphone) y la tablet.

La elección de estos dispositivos no se ha realizado aleatoriamente. Chillida (2013) ratifica, parcialmente, la selección realizada al señalar:

El Observatorio Nacional de las Telecomunicaciones y la SI (ONTSI) aporta datos del primer trimestre de 2012 sobre el equipo o dispositivo de acceso a Internet. El ordenador de sobremesa sigue siendo el principal medio utilizado para ello (68.2 por ciento de los hogares españoles con acceso a Internet), seguido de cerca por el ordenador portátil (67.8 por ciento), aunque las diferencias se van reduciendo y está a punto de cambiar el orden. El tercer medio es el teléfono móvil (45.5 por ciento), seguido por la videoconsola (9.9 por ciento) (p. 7).

Los resultados expuestos en este artículo corresponden a las respuestas otorgadas al instrumento de recogida de datos (cuestionario de naturaleza cuantitativa) que se ha aplicado a los estudiantes universitarios de cinco universidades españolas. Para seleccionar los lugares de uso de los dispositivos móviles que favorecen el aprendizaje ubicuo se ha despreciado, frente a Chillida (2013) el ordenador fijo por no considerarlo terminal que facilite, por su inmovilidad, el aprendizaje ubicuo. También se ha desechado la videoconsola. El motivo por el que se ha descartado la videoconsola es por no perseguir, a juicio de las autoras del artículo, los fines formativos en los que se centra el proyecto de investigación del que se extrae este artículo.

La muestra que ha tomado parte en la investigación, por su edad cronológica, está compuesta por nativos digitales. Fueyo (2011, p.25) nos explica cómo «se ha dado en llamar nativos digitales a las personas que por su edad tienen un dominio natural de los lenguajes digitales que utilizan las TIC». Igualmente, por la formación académica que han recibido en su etapa escolar los universitarios que componen la muestra de este estudio son alfabetos digitales, como expone González (2012, p.19) «...saben usar las tecnologías y dispositivos de forma instrumental, identificando este concepto con las competencias tecnológicas o informáticas».

Una de las conclusiones de este proyecto de investigación permite afirmar que no todos los universitarios, nativos y alfabetizados digitalmente, que han tomado parte en la investigación adquieren su formación universitaria a través de aprendizaje ubicuo. Extrapolando los resultados obtenidos al conjunto de universitarios del país podría afirmarse que no todos los universitarios en España utilizan los dispositivos móviles con fines formativos. La razón hay que buscarla en que algunos de los actuales universitarios aunque están capacitados para usar los dispositivos móviles porque en su etapa escolar han adquirido competencias instrumentales que les permiten manejar estos medios tecnológicos (están alfabetizados 
tecnológicamente), adolecen de las competencias necesarias para desenvolverse autónomamente en la sociedad del conocimiento.

Estos universitarios no constituyen el grupo de nuevos aprendices. Continúan siendo antiguos aprendices que no han conseguido desarrollar suficientemente una alfabetización multimodal o multimedia. La alfabetización tecnológica, cuando no es meramente instrumental, conlleva la adquisición de competencias más complejas y relevantes para el desarrollo del aprendizaje a través de la red, Ortega (2009, p. 6) nos indica como «la característica más relevante de la alfabetización tecnológica es su multiplicidad, la alfabetización tecnológica es múltiple porque conlleva una alfabetización instrumental, comunicativa, social, emocional y educativa, así como, el desarrollo de habilidades, destrezas y aptitudes».

\section{Los nuevos aprendices.}

Los nuevos aprendices se caracterizan por estar altamente cualificados en la multitarea. También se caracterizan porque no piensan de forma lineal y muestran variaciones en los estilos de aprendizaje (McLester, 2007; OECD, 2008; Pedró, 2006). Estos nuevos aprendices forman parte de una generación que piensa y aprende de manera interactiva (Ramos Elizondo, Herrera Bernal \& Ramírez Montoya, 2010). Los nuevos aprendices tienen la capacidad de generar contenido individualmente, a la vez que presentan mayor control y capacidad de crear que los antiguos aprendices.

Los nuevos aprendices disfrutan de un fuerte sentido de identidad y propiedad (Siemens, 2006). Tienden hacia la independencia y autonomía en sus estilos de aprendizaje, selec- cionando, entre múltiples fuentes y recursos, nuevos contenidos que se pueden combinar (Baird \& Fisher, 2006).

Los actuales estudiantes universitarios, en su mayoría, son nuevos aprendices por lo que diseñan sus estrategias formativas huyendo de la búsqueda de datos concretos (McLester, 2007). Basan sus aprendizajes en la exploración, consulta y síntesis de conocimientos más que en la asimilación de unos contenidos ofrecidos por una única fuente de conocimiento validado como es el proveniente de un libro o un profesor que imparte conferencias (Dede, 2005). No obstante, no todos los universitarios de España son capaces de generar un aprendizaje consciente, responsable y comprometido a través de las tecnologías móviles (Tabuenca, Verpoorten, Ternier, Westera \& Specht, 2013), es decir, no todos los universitarios de España se forman a través del u-learning.

Las distintas formas de adquirir el conocimiento y de enfrentarse a los aprendizajes generan, al menos, dos perfiles de estudiantes universitarios. Por un lado se encuentran aquéllos que han optado por formarse ubicuamente. Por otro, se encuentran los estudiantes universitarios, coetáneos de los anteriores, formados con sus mismos esquemas educativos y que teniendo las mismas posibilidades de acceso a las tecnologías digitales no optan por formarse a través de dispositivos móviles. Esta decisión instructiva es germen de la temida brecha digital formativa.

\section{Brecha digital formativa.}

Tedesco (2011, p. 36) indica como la brecha digital «refleja el desigual acceso de las personas a las instituciones y al uso de las tecnologías a través de las cuales se produce y 
se distribuyen las informaciones y los conocimientos más importantes». Raad (2011, p. 40) pone de manifiesto comola brecha digital también «cuantifica la diferencia existente entre países, sectores y personas que tienen acceso a los instrumentos y herramientas de la información y la capacidad de utilizarlos, y aquellos que no la tienen».

La brecha digital formativa es un nuevo concepto. Esta modalidad de brecha digital refleja la distancia formativa existente entre dos tipos de estudiantes, los estudiantes residentes y los estudiantes visitantes de la red (Hernández y Hernández, RamirezMartinell \& Cassany, 2013).

Los nuevos aprendices que adquieren su formación universitaria de manera ubicua con el uso de múltiples dispositivos móviles forman parte activa de la sociedad de la información y el conocimiento, estando constantemente disponible en los medios. Estos estudiantes son denominados estudiantes residentes (Hernández y Hernández, RamirezMartinell \& Cassany, 2013). Para ellos, todos los medios, momentos e instrumentos son elementos constitutivos del u-learning, es decir, los estudiantes residentes practican la cloudcomputing.

La conectividad de estos aprendices se manifiesta también en su gestión del tiempo. El tiempo de ocio puede convertirse en tiempo de aprendizaje. Este permanente y continuado tiempo de aprendizaje se lleva a cabo por la constante utilización de instrumentos de aprendizaje ubicuo. Tabuenca, Verpoorten, Ternier, Westera y Specht (2013, p. 3) indican como «existe la promesa virtual de que estos dispositivos cambian esta percepción «tiempo perdido» en «tiempo productivo»».

Los estudiantes residentes están constantemente conectados. Su conectividad describe su actitud de estar continuamente dis- ponibles, apoyándose en la prevalencia de la comunicación a través de los móviles, las redes sociales y la mensajería instantánea (Sevillano, 2009). Borrás (2013, p. 15) refleja como los estudiantes «que no saben utilizar las nuevas tecnologías se convierten en el nuevo sector de riesgo de exclusión social». Esta modalidad de estudiantes universitarios se denomina estudiantes visitantes de la red.

Los estudiantes visitantes de la redes se instruyen de manera tradicional, es decir, a través de la asistencia a clases, horas de estudio personal, intercambio de apuntes y ampliaciones bibliográficas de los contenidos ofrecidos en las aulas universitarias. No se aprovechan de las potencialidades que presentan las tecnologías como plantean Filippi, Lafuente y Bertone (2012, p. 1) con la «posibilidad de extender nuestro ámbito de estudio más allá de los límites físicos de la educación presencial».

Los universitarios visitantes de la red no pueden integrar, de manera natural, las actividades de aprendizaje en la vida diaria porque no encuentran un horario conveniente para aprender permanentemente durante todo el día (Tabuenca, Ternier \& Specht, 2013). Esta imposibilidad incrementa la brecha digital formativa existente entre los tipos de estudiantes universitarios. Unos se forman a través de un aprendizaje permanente tradicional (los universitarios visitantes) y otros lo hacen a través del aprendizaje ubicuo (los universitarios residentes).

\section{Aprendizaje ubicuo como evolución del aprendizaje permanente.}

Una de las conclusiones del Proyecto I+D MICINN EDU 2010-17420 señala que los estudiantes que sean capaces de gestionar su aprendizaje de forma ubicua tendrán mayo- 
res facilidades para adquirir sus conocimientos de manera integral que los que no lo hagan a través de esta opción formativa. Esta ventaja será consecuencia directa de una de las características constitutivas del ulearning: su flexibilidad para instruir en cualquier momento y en cualquier lugar. Esta característica marca un gran paso hacia delante en la educación al desarrollar una pedagogía transformada y transformadora en un entorno de aprendizaje ubicuo (De Castro,2012).

El aprendizaje ubicuo, para desarrollarse plenamente, requiere de una instrucción específica, esto es, de un entrenamiento teniendo en cuenta los aprendices, los dispositivos, la red, la plataforma y los recursos como elementos básicos y de diseño del aprendizaje ubicuo (Yang \& Pan, 2013).

El aprendizaje ubicuo (u-learning) puede considerarse la evolución natural del aprendizaje permanente puesto que actualmente las TIC son el entorno en el que se producen muchas de las interacciones y la comunicación que son la base del aprendizaje permanente de las personas (Adell \& Castañeda, 2010). Para que esta evolución natural del aprendizaje permanente al aprendizaje ubicuo, u-learning, cristalice y tome cuerpo es necesaria la creación en las instituciones educativas de un ecosistema virtual para la formación y la relación (Cabero, 2013). Un ecosistema que permita que la educación permanente juegue, en el nivel superior, el rol de instrumento igualitario, en el sentido de que permita ofrecer una segunda oportunidad a las personas que por razones económicas, $\mathrm{o}$ por cualquier otro motivo, no pudieron tener acceso en la edad normal a la educación superior (Tünnermann, 2010).

La noción de educación permanente también se vincula con el concepto de una sociedad del aprendizaje, durante toda la vida y significa que no sólo las personas deben aprovechar las oportunidades creadas por la sociedad, sino también que las sociedades deben planificarse para dar a sus miembros nuevas oportunidades (Nesbit, Dunlop \& Gibson, 2013).

\section{Metodología.}

\subsection{Objetivo del estudio.}

Identificar los lugares de uso de los ordenadores portátiles, los teléfonos móviles y las tablets con usos académicos en cinco universidades españolas.

\subsection{Muestra.}

La muestra que tomó parte en esta investigación estuvo constituida por 451 estudiantes pertenecientes a cinco universidades españolas (Universidad Complutense de Madrid, Granada, Oviedo, UNED y Universidad de Vigo). La muestra estudiada estaba conformada por un $76.3 \%$ de mujeres y un $23.7 \%$ de hombres.

Atendiendo a las edades de la muestra, ésta presentaba las siguientes agrupaciones: en torno a los 18 y 20 años participó el $24.4 \%$ de los casos. Entre los 21 y 23 años el 33\%. Entre los 24 y 27 años el 10\%. Entre los 28 y 31 años el $5.7 \%$. Con más de 31 años el $26.9 \%$.

Los estudios que realizaban los 451 estudiantes que tomaron parte en la investigación responden a los siguientes porcentajes: Licenciaturas $24.2 \%$ de los sujetos, Grados $70.5 \%$ de los sujetos, Doctorados $0.3 \%$ de los sujetos, Másteres $4.7 \%$ de los sujetos, Postlicenciaturas $0.3 \%$ de los sujetos que respondieron el cuestionario.

El muestreo utilizado en la investigación fue incidental. No aleatorio y por conglomerados. 


\subsection{Instrumentos de recogida de informa- ción y análisis de datos.}

La información se recogió a través de un cuestionario cuantitativo conformado por una escala tipo Likert de cinco posibles respuestas. El alfa de Cronbach del total del instrumento fue de .81 por lo que el instrumento de recogida de información puede considerarse fiable. También se utilizó la etnografía multisituada y una entrevista en profundidad. En el tratamiento y análisis de los datos se utilizaron los métodos descriptivos, inferencias estadísticas y análisis de contenido en textos. Se han analizados los datos con la técnica de estructuración de documentos textuales por medio de QDA Miner 3.24.

\section{Resultados.}

\subsection{Usos de los dispositivos en las aulas.}

Los terminales estudiados han sido el ordenador portátil, el teléfono móvil no inteli- gente, el smartphone y la tablet. Los datos que se plasman en la explicación de los resultados de este artículo proceden de las categorías «siempre» $\mathrm{y}$ «a menudo» así como de las categorías «nada» 0 «casi nada». El resto de los resultados pueden observarse en los gráficos incorporados en el artículo.

Las conclusiones obtenidas en el estudio permiten afirmar que los dos dispositivos facilitadores de aprendizaje ubicuo que se usan, mayoritariamente en las aulas, son el teléfono móvil y el ordenador portátil y el dispositivo que menos se utiliza es la tablet.

El teléfono móvil no inteligente y el smartphone se utilizan un $38.33 \%$ «siempre» o «a menudo». El ordenador portátil se utiliza en las aulas, un $21.66 \%$ entre las categorías «siempre» o «a menudo». No todos los estudiantes utilizan estos dispositivos. Un 57\% no utiliza «nada o «casi nada» el portátil en las aulas. La tablet tampoco la utiliza «nada» en las aulas un $93.4 \%$ de los universitarios en España. (Véase Gráfico 1).

Los datos obtenidos permiten concluir que el dispositivo facilitador de u-learning que

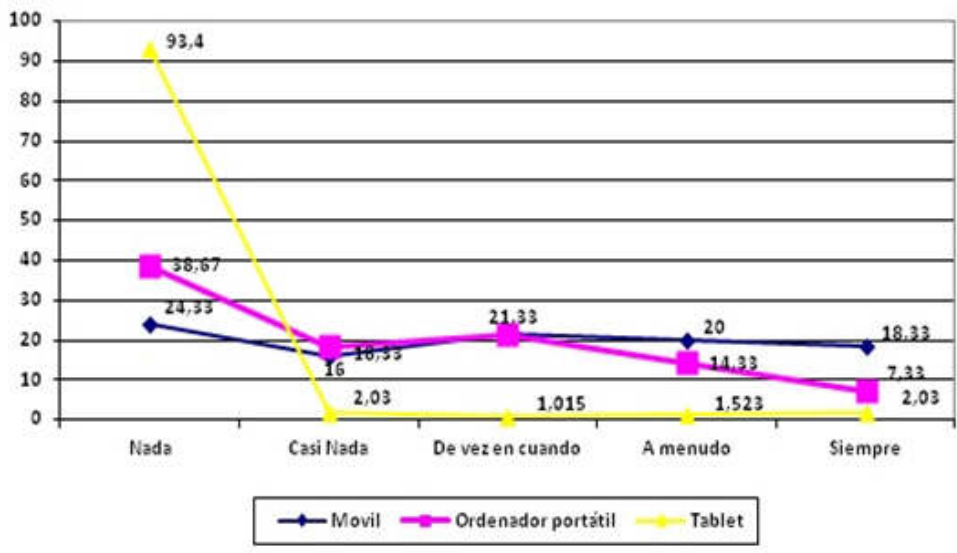

Gráfico 1. Uso del ordenador portátil, el teléfono móvil no inteligente y smartphone y la tablet en las aulas.

Píxel-Bit. Revista de Medios y Educación. No 46. Enero 2015. ISSN: 1133-8482. e-ISSN: 2171-7966. doi: http://dx.doi.org/10.12795/pixelbit.2015.i46.10 


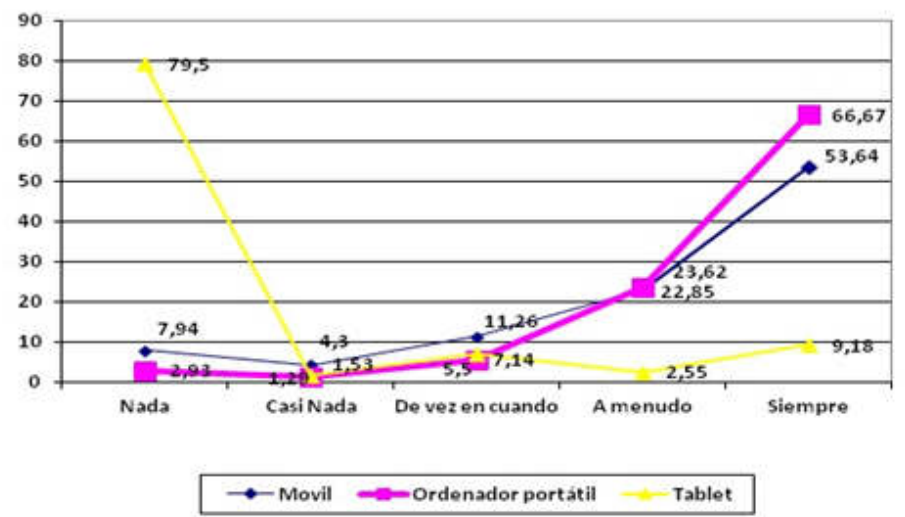

Gráfico 2.Uso del ordenador portátil, el teléfono móvil no inteligente y smartphone y la tablet en los domicilios.

mayoritariamente se usa en las aulas universitarias en España, entre la muestra estudiada, es el teléfono móvil en su versión no inteligente y smartphone y el dispositivo menos utilizado la tablet.

\subsection{Usos de los dispositivos en los domici- lios.}

Los dispositivos facilitadores de u-learning que más se utilizan en el domicilio son, por este orden, el ordenador portátil y el teléfono móvil no inteligente y smartphone. El dispositivo que menos se utiliza es la tablet.

El ordenador portátilse utiliza en los domicilios un $90.29 \%$ «siempre» o «a menudo». Los teléfonos móviles no inteligentes y smartphone se utilizan un $74.49 \%$ «siempre» o «a menudo» mientras que la tablet es el dispositivo menos utilizado en los domicilios conun $79.5 \%$ de respuestas en la categoría de frecuencia de uso «nada» (Véase Gráfico 2).

Los datos obtenidos permiten concluir que el dispositivo facilitador de u-learning que mayoritariamente se usa en los domicilios en España entre la muestra estudiada es el ordenador portátil y el dispositivo menos utilizado la tablet.

\subsection{Usos de los dispositivos en el lugar de trabajo.}

No está claro qué dispositivos de u-learning utilizan, en los lugares de trabajo, los estudiantes universitarios que han tomado parte en el estudio. Los datos obtenidos indican que el ordenador portátil es utilizado en el trabajo un $40.54 \%$ entre las categorías «Siempre» $\mathrm{y}$ «A menudo». Sin embargo, un $41.76 \%$ de la muestra manifiesta que no lo utiliza «Nada» 0 «Casi Nada». Igual ambivalencia se produce con los datos centrados en el teléfono móvil no inteligente y el smartphone. Los teléfonos móviles son utilizados un $39,08 \%$ «siempre y «a menudo» y no se utiliza «nada» o «casi nada» un 40,23\% de los casos. La tablet no se utiliza «nada» en el 91.71\% (Véase Gráfico 3). 


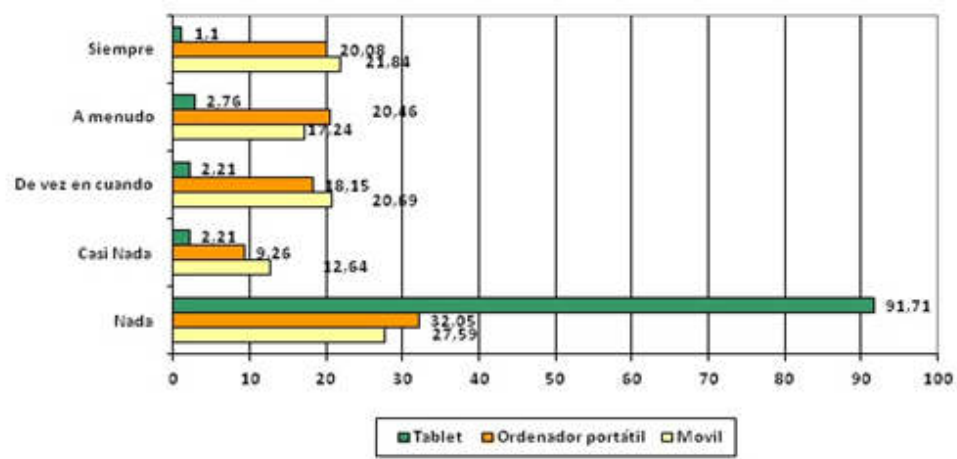

Gráfico 3. Uso del ordenador portátil, el teléfono móvil no inteligente y smartphone y la tablet en lugar de trabajo.

\subsection{Usos de los dispositivos en la bibliote-} ca.

Los resultados de frecuencia de uso de dispositivos móviles con fines formativos en la biblioteca eran predecibles. Los nuevos aprendices no suelen utilizar las bibliotecas. Estos espacios formativos se ocupan, con mayor frecuencia por antiguos aprendices que siguen formándose con libros impresos, papel y bolígrafos. En el estudio realizado se observó que el único dispositivo facilitador de aprendizaje ubicuo usado en las bibliotecas era el ordenador portátil.

Los datos obtenidos fueron: el ordenador portátil se utiliza un $33.22 \%$ en la biblioteca entre las categorías «siempre» o «a menudo». No obstante, un $41.86 \%$ de los estudiantes universitarios manifiesta no utilizarlos

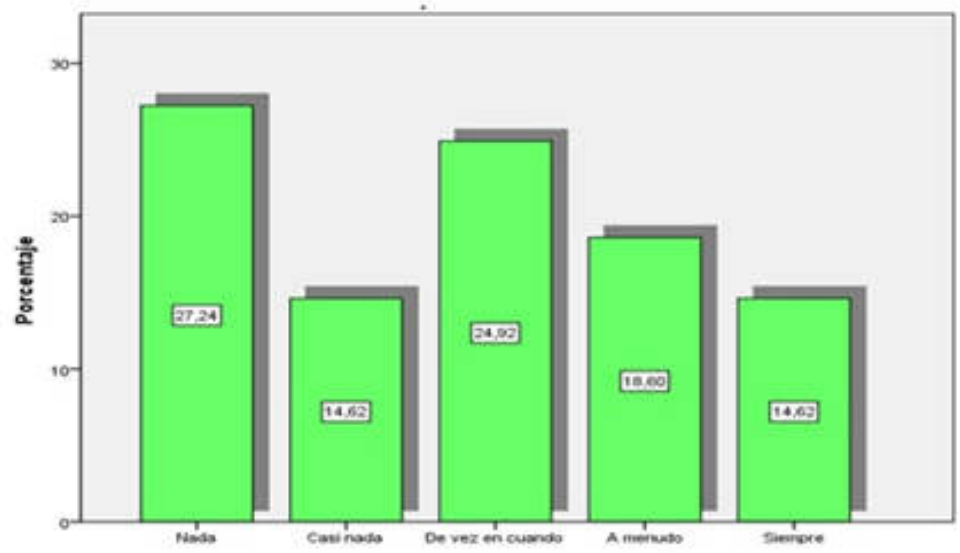

Gráfico 4.Uso del ordenador portátil en la biblioteca. 


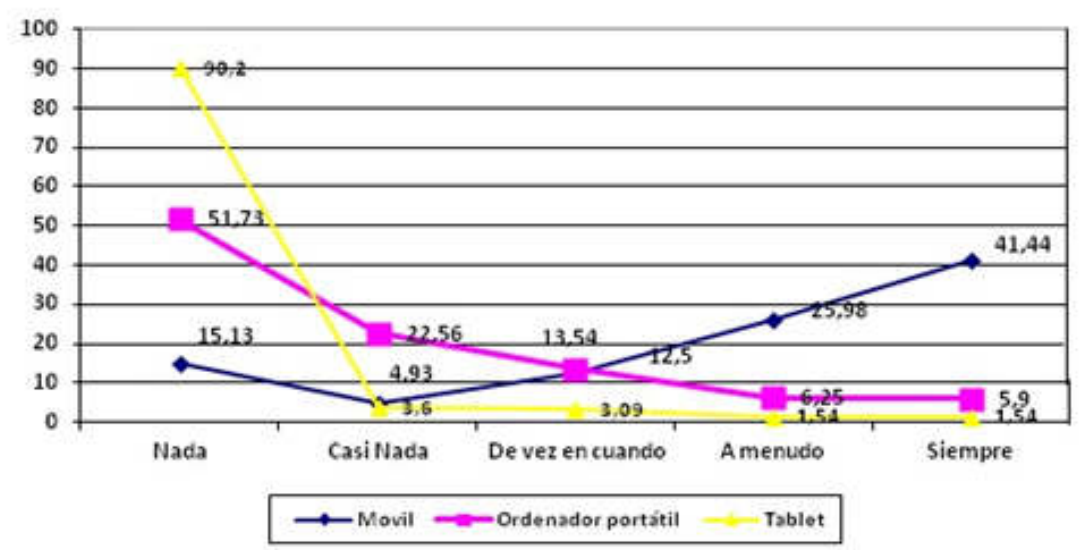

Gráfico 5.Uso del teléfono móvil no inteligente y smartphone, ordenador portátil y tablet en las zonas de ocio.

«nada» o «casi nada» y un $24.92 \%$ de los estudiantes lo utilizan solo «de vez en cuando» (Véase Gráfico 4).

\subsection{Usos de los dispositivos en las zonas de ocio.}

El teléfono móvil no inteligente y Smartphone es utilizado un $77.42 \%$ «siempre» $\mathrm{y}$ «a menudo» en las zonas de ocio entre los universitarios que han tomado parte en el estudio. A este dispositivo le sigue, en frecuencia de uso, el ordenador portátil con un porcentaje de $12.15 \%$ entre las categorías «siempre» $\mathrm{y}$ «a menudo» aunque el $74.29 \%$ de los universitarios no lo utilice «nada o «casi nada». La tablet no se utiliza «nada», un $90.2 \%$ (Véase Gráfico 5). Los resultados obtenidos muestran, claramente, que el dispositivo más utilizado en las zonas de ocio es el teléfono móvil no inteligente y Smartphone y el dispositivo menos utilizado es la Tablet.

Otros datos obtenidos en los grupos de discusión de los que no se ofrecen resultados en este artículo muestran que el portátil no se utiliza en la calle ( $92.11 \%$ de los casos), ni en los medios de transportes $(3.75 \%)$ de los casos. En contraposición, el teléfono móvil sí se utiliza en la calle $(65.67 \%$ de los casos) así como en los medios de transportes (59.93\%de los casos).

\section{Conclusiones.}

Los estudiantes universitarios que han tomado parte en el estudio o bien son nativos digitales o bien son alfabetos digitales. Estas circunstancias iniciales no garantizan que todos utilicen como metodología formativa, en el nivel universitario, el aprendizaje ubicuo. Los que utilizan el u-learning constituyen el grupo de los nuevos aprendices. Un grupo de ellos puede considerarse también estudiantes residentes.

La universidad también acoge a otro grupo de estudiantes que partiendo de la misma situación instructiva en la escuela y el instituto que los nuevos aprendices, en el nivel universitario, siguen formándose a través de 
la metodología analógica tradicional. Estos son los antiguos aprendices y, entre ellos se encuentran los estudiantes visitantes de la red.

Entre ambas agrupaciones estudiantiles se abre una nueva modalidad de brecha digital, la brecha digital formativa que se manifiesta fundamentalmente en el uso que otorgan, unos y otros a los dispositivos móviles para desarrollar su aprendizaje ubicuo. El tamaño de la brecha digital formativa justifica la diversidad de puntuaciones encontrada al cuantificar el uso que se les otorgan a los dispositivos móviles facilitadores de aprendizaje ubicuo en distintas ubicaciones físicas.

Quiere entenderse que los estudiantes residentes son quienes los utilizan, con fines formativos, mayoritariamente el ordenador portátil en las aulas, los domicilios, los lugares de trabajo y las bibliotecas. Estos mismos estudiantes son los que utilizan mayoritariamente en los lugares de recreo los smartphones. Los estudiantes visitantes de la red son los que menos usan los mismos dispositivos en los mismos espacios.

Un dato común a ambos tipos de estudiantes es el uso infrecuente, con fines formativos, de la Tablet. Las razones de este uso restrictivo pueden ser variadas: relación precio-utilidad del dispositivo, dificultad para el tratamiento de textos, tamaño de la pantalla, entre otros.Con independencia del motivo por el que se opte en la discusión de los resultados, la realidad es que «los equipos portátiles son el tipo de dispositivo móvil más utilizado. Estudios recientes han encontrado resultados similares argumentando motivos ergonómicos. Los estudiantes prefieren pantallas más grandes en comparación a Smartphone o tabletas» (Tapellini, 2011, citado en Tabuenca, Ternier \& Specht, 2013, p.3).

El ordenador portátil, es por tanto, el dispositivo más utilizado. Se usa, mayoritariamente, en los domicilios. Le sigue por frecuencia de uso el lugar de trabajo. El tercer lugar lo ocupan las bibliotecas. Las aulas universitarias son el cuarto ámbito de uso y las zonas de ocio, transportes y calle el último.

Los resultados de la investigación muestran que los dispositivos móviles utilizados con más asiduidad, como recursos formativos, son aquellos útiles para la realización de trabajos educativos, como por ejemplo, el ordenador portátil. En las aulas no se usan los dispositivos móviles porque se fomenta la metodología tradicional que conlleva el uso de recursos no tecnológicos.

Otra conclusión relevante de esta investigación es que las bibliotecas, entendidas como dinamizadoras de aprendizaje, deberían de ofrecer el uso de dispositivos móviles a través del préstamo y una formación específica en aprendizaje ubicuo para la utilización formativa de estos nuevos dispositivos que son facilitadores de la investigación y el aprendizaje virtual.

Los resultados exponen la falta de una alfabetización digital y una formación ubicua de los estudiantes y el analfabetismo digital del profesorado para el desarrollo de estrategias metodológicas que guíen a los alumnos en un aprendizaje ubicuo facilitador de una formación universitaria.

Los análisis realizados llevan a concluir la necesidad de que las entidades educativas maximicen los recursos tecnológicos educativos a través de la utilización adecuada de los dispositivos móviles, para mejorar los procesos de enseñanza-aprendizaje. La información, comunicación y formación que se obtiene a través de los dispositivos móviles es muy amplia y necesaria para optimizar el tiempo y mejorar el aprendizaje. Por lo tanto, es indispensable la adquisición de una nueva

Píxel-Bit. Revista de Medios y Educación. No 46. Enero 2015. ISSN: 1133-8482. e-ISSN: 2171-7966. doi: http://dx.doi.org/10.12795/pixelbit.2015.i46.10 
alfabetización digital formativa, la alfabetización digital ubicua.

\section{Fuentes de financiación.}

La presente investigación forma parte del Proyecto I+D MICINN EDU 2010-17420, denominado Aprendizaje ubicuo con dispositivos móviles: elaboración y desarrollo de un mapa de competencias en educación superior dirigido por la Dra. María Luisa Sevillano García.

\section{Referencias bibliográficas.}

Adell Segura, J. \& Castañeda Quintero, L. (2010). Los Entornos Personales de Aprendizaje (PLEs): una nueva manera de entender el aprendizaje. En R. Roig Vila \& M. Fiorucci (Eds.).Claves para la investigación en innovación y calidad educativas. La integración de las Tecnologías de la Información y la Comunicación y la Interculturalidad en las aulas. Stumenti di ricerca per l'innovaziones e la qualità in ámbito educativo. La Tecnologie dell'informazione e della Comunicaziones e l'interculturalità nella scuola.Alcoy: Marfil Roma TRE Universita degli studi. Recuperado de http://digitum.um.es/jspui/bitstream/ 10201/17247/1/Ade11\%26Casta\%C3\% B1eda_2010.pdf

Baird, D.E. \& Fisher, M. (2006). Neomillennial User Experience Design Strategies: Utilizing Social Networking Media to Support 'Always On' Learning Styles. Journal of Educational Technology Systems, 34 (1), 5-32.doi: 10.2190/6WMW-47L0-M81Q12G1

Borrás Gené, O. (2013). Comunidades virtuales.Recuperado de http://oa.upm.es/ 14504/1/Curso_introduccion_redes_ sociales.pdf
Cabero Almenara, J. (2013). El aprendizaje autorregulado como marco teórico para la aplicación educativa de las comunidades virtuales y los entornos personales de aprendizaje. Revista Teoría de la Educación: Educación y Cultura en la Sociedad de la Información,14 (2), 133-156. Recuperado de http:/ /campus.usal.es/ revistas_trabajo/ index.php/revistatesi/article/view/10217/ 10626

Chillida, J. R. (2013). ¿Hay brecha digital en los hogares españoles?: Banda Ancha. Telos: Cuadernos de comunicación e innovación, 94, 6-8.

De Castro Lozano, C. (2012). El futuro de las tecnologías digitales aplicadas al aprendizaje de personas con necesidades educativas especiales. RED, Revista de Educación a Distancia, 32, 1-43. Recuperado de http:// www.um.es/ead/red/32

Dede, C. (2005). Planning for Neomillennial Learning Styles. Educause Quarterly, 28(1), 7-12. Recuperado de http://www.educause. edu/pub/eq/eqm05/eqm0511.asp

Filippi, J. L., Lafuente, G. J.\& Bertone, R. A. (2012). Diseño de un ambiente de aprendizaje colaborativo. $V$ Congreso de Tecnología en Educación y Educación en Tecnología. Red de Universidades con Carreras en Informática (RedUNCI).Recuperado de http:// hdl.handle.net/10915/18477

Fueyo Gutiérrez, M.A. (2011). Comunicación y educación en los nuevos entornos: ¿Nativos o cautivos digitales?, Ábaco, 2(6869), 22-28. Recuperado de http:// www.cinelatinoamericano.org/assets/docs/ 0499Ed01.pdf

González Fernández-Villavicencio, N. (2012). Alfabetización para una cultura social, digital, mediática y en red. Revista Española de Documentación Científica, 35, 17-45. doi: 10.3989/redc.2012.mono.976. 
Hernández y Hernández, D., RamirezMartinell, A. \& Cassany, D. (2013). Categorizando a los usuarios de sistemas digitales. Píxel-Bit, Revista de medios y educación, 44, 113-126. doi: http://dx.doi.org/ 10.12795/pixelbit.2014.i44.08

McLester, S. (2007). Technology Literacy and the MySpace Generation: They're Not Asking Permission. Technology \& Learning, 27(8), 16-22.

Nesbit, T., Dunlop, C.\& Gibson, L. (2013). Lifelong learning in institutions of higher education. Canadian Journal of University Continuing Education, 33 (1), 35-60. Recuperado de http://ejournals.library.ualberta.ca/ index.php/cjuce-rcepu/article/view/19944/ 15461

OECD (2008). New Millennium Learners: Initial findings on the effects of digital technologies on school-age learners. OECD \& CERI. Recuperado de http://www.oecd.org/ dataoecd/39/51/40554230.pdf

Ortega Sánchez, I. (2009). Alfabetización tecnológica. Teoría de la educación. Educación y cultura en la sociedad de la información, 10 (2), 5-7.Recuperado dehttp:// www.usal.es/ teoriaeducacion/rev_n umero_10_02/n10_02_priegue.pdf

Pedró, F. (2006). The new Millennium Learners: Challenging our Views on ICT and Learning. OECD \& CERI. Recuperado de http://www.oecd.org/dataoecd/1/1/ 38358359.pdf

Raad, A. M. (2011). Exclusión digital: nuevas caras de viejos malestares. Revista Mad, 14, 40-46. doi: 10.5354/0718-0527.2006.14203.

Ramos Elizondo, A. I., Herrera Bernal, J. A. \& Ramírez Montoya, M. S. (2010). Desarrollo de habilidades cognitivas con aprendizaje móvil: Un estudio de casos. Revista Comunicar, XVII (34), 201-209. doi: 10.3916/C342010-03-20.
Sevillano García, Mª.L. (2009) (Ed.). Competencias para el uso de herramientas virtuales en la vida, trabajo y formación permanentes. Madrid: Pearson.

Siemens, G. (2006). Knowing Knowledge. Recuperado de

http:// www.knowingknowledge.com

Tabuenca, B., Verpoorten, D., Ternier, S., Westera, W.\& Specht, M. (2013). Fomento de la práctica reflexiva sobre el aprendizaje mediante el uso de tecnologías móviles. RED. Revista de Educación a Distancia, 37, 1-14. Recuperado de http://www.um.es/ead/red/37/ tabuenca2.pdf

Tabuenca, B., Ternier, S. \& Specht, M. (2013). Patrones cotidianos en estudiantes de formación continua para la creación de ecologías de aprendizaje. RED, Revista de Educación a Distancia, 37. Recuperado de http://www.um.es/ead/red/37

Tedesco, J. C. (2011). Los desafíos de la educación básica en el siglo XXI. Revista Iberoamericana de Educación, 55 (1), 31-47. Recuperado de http://www.rieoei.org/ rie55a01.pdf

Tünnermann Bernheim, C. (2010). La educación permanente y su impacto en la educación superior. Revista Iberoamericana de Educación Superior (RIES), 1 (1),120-133. Recuperado de http://ries.universia.net/ index.php/ries/article/view/25/ educacion_permanente

Yang, X. \& Pan, F. (2013). A Mode Design Research On Ubiquitous Learning. In Proceedings of the 2013 International Conference on Information, Business and Education Technology (ICIBET 2013). Atlantis Press. (1210-1213). doi:10.2991/ icibet.2013.228.

Fecha de recepción: 26-05-2014 Fecha de evaluación: 15-09-2014 Fecha de aceptación: 20-10-2014 